\title{
Association of a 20-IncRNA signature with mutation load of colon adenocarcinoma and immune microenvironment
}

Yan Lin ( $\sim$ linyan@stu.gxmu.edu.cn )

Guangxi Cancer Hospital and Guangxi Medical University Affiliated Cancer Hospital https://orcid.org/0000-0003-0343-3277

Qian Li

Guangxi Cancer Hospital and Guangxi Medical University Affiliated Cancer Hospital

Ziyu Liu

Guangxi Medical University

Rong Liang

Guangxi Cancer Hospital and Guangxi Medical University Affiliated Cancer Hospital

Jiazhou Ye

Guangxi Cancer Hospital and Guangxi Medical University Affiliated Cancer Hospital

\section{Ziqin He}

Guangxi Medical University

\section{Xing Gao}

Guangxi Cancer Hospital and Guangxi Medical University Affiliated Cancer Hospital Jinyan Zhang

Guangxi Cancer Hospital and Guangxi Medical University Affiliated Cancer Hospital

Min Luo

Guangxi Cancer Hospital and Guangxi Medical University Affiliated Cancer Hospital

\section{Yongqiang Li}

Guangxi Cancer Hospital and Guangxi Medical University Affiliated Cancer Hospital

\section{Lianying Ge}

Guangxi Cancer Hospital and Guangxi Medical University Affiliated Cancer Hospital

\section{Research Article}

Keywords: Colon adenocarcinoma, tumor mutation burden, IncRNA, immunotherapy, LASSO

Posted Date: January 12th, 2022

DOI: https://doi.org/10.21203/rs.3.rs-1204020/v1 
License: (c) (i) This work is licensed under a Creative Commons Attribution 4.0 International License. Read Full License 


\section{Abstract \\ Purpose}

Tumor mutation burden (TMB) is an emerging biomarker for predicting immune checkpoint inhibitors (ICI). However, the role of TMB associated with long non-coding RNAs (IncRNAs) expression was not clear for preexisting immunity in colon adenocarcinoma (COAD).

\section{Methods}

The mutation data for COAD was downloaded from the Cancer Genome atlas (TCGA) database. A set of differentially expressed IncRNAs (DE IncRNAs) were identified between high or low TMB colon adenocarcinoma samples through differential expression analysis. The absolute shrinkage and selection operator (LASSO) method was used to define a subset of the DE IncRNAs as a "signature" for predicting TMB levels in the training cohort. After validating the signature on a test cohort, its potential correlations with the indicators of the efficacy of anti-immune checkpoint therapy were explored. Gene set variation analysis (GSVA) was carried out to explore the biological functions between high and low signature DE IncRNAs sample groups.

\section{Results}

A signature comprising $20 \mathrm{DE}$ IncRNAs predicted TMB levels was identified by lasso method. And the model was with high accuracy in the training cohort $(A \cup C=0.92)$ and test cohort ( $A U C=0.93)$. Moreover, the signature was also associated with T-effector and interferon-y gene signature, expression of PDCD1, CD274, CTLA4, as well as abundance of TILs. Tumors receiving a low score for the 20-IncRNA signature received a high GSVA score for multiple immune-related pathways.

\section{Conclusions}

The 20-IncRNA signature can accurately predict TMB in colon adenocarcinoma and is associated with known indicators of immune checkpoint inhibitor efficacy and preexisting immunity.

\section{Introduction}

Colon adenocarcinoma (COAD) is common worldwide and is associated with significant mortality in late stages $^{[1]}$. Although early diagnosis and therapeutic strategies have been well established for COAD patient invasion, metastasis and recurrence of the disease are still challenging. Currently, immunotherapies, such as those involving immune checkpoint inhibitors (ICls), can greatly improve 
prognosis in patients with colon adenocarcinoma ${ }^{[2,3]}$. Identifying biomarkers that predict the efficacy of such therapies would help clinicians personalize treatment.

As the backbone of modern cancer treatment, immuno-oncology is revolutionizing cancer treatment. Immune checkpoint inhibitors (ICl) work by releasing brakes for antitumor response of the immune system ${ }^{[4]}$. Although $\mathrm{ICl}$ therapy has made breakthrough progress, it is not without side effects. And there was not an ideal curative effect in all patients treated with ICl therapy. Therefore, it is urgent to find a biomarker that can identify a good response to ICI therapy. The programmed death ligand 1 (PD-L1) and microsatellites instability high (MSI-H) or mismatch repair deficiency (dMMR) are used clinically as biomarkers currently. Although both PD-L1 and MSI-H/dMMR have identified as predictors of ICl therapy response. However, they are still not perfect, and their sensitivity and specificity are not good ${ }^{[5]}$. Therefore, it is necessary to continue to seek better biomarkers for predicting the response to ICI therapy. One of the emerging biomarkers is tumor mutation burden (TMB), defined as the number of somatic mutations in a defined region of a tumor genome ${ }^{[6]}$. For example, tumors with high TMB may exhibit specific genetic alterations associated with $\mathrm{ICl}$ efficacy ${ }^{[7]}$. However, TMB is currently determined using expensive wholeexome sequencing, which limits its widespread use in the clinic.

Long noncoding RNAs (IncRNAs), which are noncoding RNAs more than 200 nucleotides long, play important roles in various immune processes. And IncRNA can be used as a potential prognostic molecular biomarker for lung cancer ${ }^{[8]}$. In this study, we hypothesized that they might be associated with TMB and therefore serve as an inexpensive biomarker for predicting the efficacy of immunotherapy against colon adenocarcinoma. We screened a public database of colon adenocarcinomas for IncRNAs whose expression differed significantly between tumors with low or high TMB. From these differentially expressed IncRNAs, we constructed a 20-IncRNA signature that accurately predicted TMB and was associated with several known indicators of ICl efficacy.

\section{Methods}

Detailed methods are described in Supplementary Materials and Methods .

\section{Statistical analysis}

All analyses were performed using R (version 3.6.1, http://www.r-project.org/). Differences in categorical data were assessed for significance using the $\chi 2$ test; differences in continuous data were assessed using the unpaired t-test. ROC curves were drawn and compared using the pROC package (34) in R. Unless otherwise stated, $P=0.05$ was taken as the threshold of significance.

\section{Results}

Differences in IncRNA expression between colon adenocarcinoma tumors with low or high TMB 
Clinicopathological characteristics of patients and their colon adenocarcinoma samples did not differ significantly between the training and test cohorts (Table 1). A total of 326 DE IncRNAs were identified, including 100 that were up-regulated and 226 that were down-regulated in tumors with high TMB relative to tumors with low TMB in the training set (Figure 1A). Hierarchical clustering showed that DE IncRNAs that were differentially expressed to the greatest extent could distinguish low and high TMB (Figure 1B), and this was supported by principal component analysis (Figure 1C).

Using the LASSO method and 10-fold cross validation, 20 IncRNAs were identified with non-zero regression coefficients (Figure 1D). These were incorporated into the following scoring formula: Score $=$ $(0.08653188$ * AL117382.2) + (-0.1327067 * LINC02446) + 0.23340405 * LINC005258 + (-0.1271361 * LINC02195区 $+(0.03709934 *$ AC239584.1) $+(0.04032968 *$ C027348.1 $)+(-0.0608802 *$ TFAP2A-AS1 $)+$

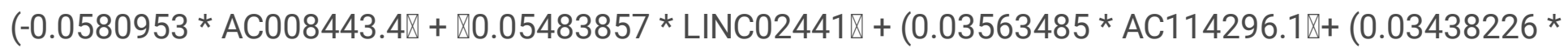
$A C 123023.1)+(0.08208376 * A L 354861.3)+(-0.0191279 * A L 022316.1 \otimes+(-0.1453998 * A P 001099.18+$ $(-0.0273567$ * LINC01630区 $+(0.07992558$ * AC073349.1区 + $(-0.002779 *$ HOXC-AS2 $)+(-0.0058216$ * AC129492.2) + (-0.0793168* LINC02620 $+(-0.1369001 * A C 092813.1) .$. When classifying TMB as low or high, this scoring formula showed accuracies of 0.92 in the training cohort, 0.93 in the test cohort, and 0.93 in the two cohorts combined. The formula also showed good accuracy based on Se, Sp, PPV, and NPV (Table 2). The area under ROC curves was 0.997 in the training cohort, 0.967 in the test cohort, and 0.989 in both cohorts combined (Figure 1E-F).

\section{Nomogram development}

To predict the recurrence probability of patients with COAD using a quantitative method, we constructed a nomogram that integrated both the 10-IncRNA-based signature, TMB and the conventional clinicopathological factors (Figure 2A) to predict 3- and 5-year OS probabilities. Calibration plots indicated that the nomogram had good accuracy as an ideal model both (Figure 2B-C). In addition, we found that patients with high-expression AC027348.1 ( $P=0.0499), A C 123023.1(P=0.022)$ and AC073349.1 ( $P=0.021)$ were more likely to have death due to COAD. However, the patients with lowexpression LINC02195 ( $P=0.031)$, TFAP2A-AS1 $(P=0.011)$, AL354861.3 $(P=0.019)$ and LINC01630 $(P=0.018)$ had higher survival rates. (Figure 2D-J).

The 20-IncRNA score correlated positively with abundance of resting natural killer (NK) cells and negatively with abundance of activated dendritic cells, M1 macrophages, activated NK cells, CD8+ T cell and $\mathrm{T}$ regulatory cells (Tregs) (Figure $2 \mathrm{~K}$ ). It did not correlate with abundance of plasma cells, endothelial cells, fibroblasts or CD4+ T cells. The various IncRNAs in the signature correlated with TIL abundances to quite different extents. For example, levels of AL117382.2 showed a strong negative correlation with abundances of resting mast cells, regulatory $T$ cells (Tregs), activated NK cells and activated dendritic cells. Conversely, levels of LINC02446 showed a strong positive correlation with activated NK cells, M1 macrophages and CD8+ T cells (Figure $2 \mathrm{~L}$ ). These results suggest that the IncRNA signature score can reflect patterns of immune invasion of colon adenocarcinoma tumors.

\section{Correlation between 20-IncRNA signature score and indicators of ICl efficacy}


The score correlated negatively with the following indicators: TMB $(r=-058)$, T-effector and interferon- $\gamma$ gene signature $(r=-0.54)$, as well as expression of PDCD1 $(r=-0.49)$, CD274 $(r=-0.61)$ and CTLA4 $(R=$ -0.48) (all $P<0.001$, Figure 3A-E). Samples with low scores on the 20 -IncRNA signature showed high GSVA scores on several immune-related pathways, including "natural killer cell mediated cytotoxicity", "intestinal immune network for IgA production", "primary immunodeficiency", and "Toll-like receptor signaling". In contrast, samples with high scores on the 20-IncRNA signature showed high GSVA scores on several metabolic pathways, including "steroid biosynthesis", "selenoamino acid metabolism", "nicotinate and nicotinamide metabolism", and "glycosylphosphatidylinositol anchor biosynthesis". These results suggested that patients with tumors scoring low on the 20-IncRNA signature mount a substantial immune response to cancer (Figure 3F).

\section{The signature IncRNA interacts with proteins encoded by genes in the VEGF/MAPK signaling pathway to improve ICl therapy}

We selected VEGF and MAPK signaling pathways for further exploration, two classical immune-related signaling pathways. Based on the RNAInter database, we found that there were a wide range of interactions among 5 signature IncRNAs (AC008443.4, AC073349.1, AC239584.1, LINC00525 and AL0223161.1) with the proteins encoded by genes in VEGF/MAPK (Figure 4A). And we found that the interaction probability was higher than 0.5 between IncRNA and protein, indicating the signature IncRNA and protein were likely to interact with each other (Table 3). Compared with normal tissues, 3 characterized IncRNAs (AC008443.4, AL0223161.1, LINC00525) interacting proteins (Prediction using RF classifier冈0.8, Prediction using SVM classifierख0.9) exhibited high staining in tumor tissues (Figure 4B). Molecular docking indicated that characterized IncRNAs had the potential to target binding proteins (Figure 4C). Besides, Prognostic prediction of targeted proteins in the validation set, high-expression EGFR $(P=0.0013), \operatorname{HSPA6}(P=0.029)$, SOS1 $(P=0.018)$, TNF $(P=0.004)$, VEGFA $(P<0.0001)$ and MAP3K4 $(P<0.0001)$ had poor survival (Figure $S 1)$. Furthermore, we depicted a proposed mechanism based on the signature IncRNA-protein. As shown in Figure 4D, the COAD patients with the low signature score may receive better immunotherapy efficiency with $\mathrm{ICI}$ therapy.

\section{Drug response characteristics based on TMB related DE IncRNAs}

We trained a predictive model against GDSC data in order to estimate the IC50 for each sample in the $\mathrm{TMB}^{\text {high }}$ and $\mathrm{TMB}^{\text {low }}$ groups. We predicted 12 drugs that might affect genes in the TMB ${ }^{\text {high }}$ and $\mathrm{TMB}^{\text {low }}$ groups (Figure 5): ZM.447439, CCT018159, KU.55933, BAY.61.3606, Cisplatin, AUY922, AICAR, TW.37, Camptothecin, W02009093972, X17.AAG and Thapsigargin.

\section{Discussion}

Colon adenocarcinoma is one of the most frequent malignancies worldwide, causing about 694,000 deaths every year ${ }^{[9]}$. It takes approximately 5-10 years for the normal mucosa to transform into an adenomatous polyp and then into invasive adenocarcinoma ${ }^{[10]}$. Five-year survival rates are $90 \%$ for 
patients with tumors in TNM stage I, 80\% in TNM stage II, 60\% in TNM stage III, and 8\% in TNM stage $\mathrm{IV}^{[11]}$. Immunotherapy can be effective against the disease, as well as against multiple tumors. To help identify patients more likely to respond to such therapy, we developed a model based on IncRNA expression levels to predict TMB in colon adenocarcinoma. The model was trained in one cohort and then validated in an independent one, and it showed good performance based on Se, Sp, PPV, NPV and area under the ROC curve.

Functional enrichment analysis showed that the DE IncRNAs in the signature are involved in several immune-related pathways, including antigen processing and presentation ${ }^{[12]}$, peroxisomes ${ }^{[13]}$, and NK cell-mediated cytotoxicity ${ }^{[14]}$. The score for the 20 -IncRNA signature correlated strongly with TMB, which may be a biomarker of the efficacy of immunotherapy ${ }^{[15]}$. In ovarian tumors expressing mutated BRCA$1 / 2$, high TMB as well as abundance of TILs correlate with improved overall survival ${ }^{[16,17]}$.The reasons for this should be explored further.

We did find, however, that scores for the 20-IncRNA signature correlated with markers of numerous TILs associated with immune response to cancer ${ }^{[18]}$. Scores correlated positively with resting NK cell abundance, and chemotherapy can strengthen the patient's antitumor immune response by reducing the number of activated NK TILs ${ }^{[19]}$. Therefore, patients with low IncRNA-based scores may mount a stronger immune response to colon adenocarcinoma.

In contrast, the score of the 20-IncRNA signature did not correlate with the abundance of plasma or CD4+ memory $T$ cells, which should be further explored. B cells can differentiate into plasma cells upon antigen activation, contributing to humoral immunity. CD4+ memory $T$ cells can suppress the outgrowth of tumor cells $^{[20]}$ over long periods ${ }^{[21]}$.

Patients with high scores on the 20-IncRNA signature may show AL117382.2 up-expression, which is associated with reduced abundance of Tregs, activated NK cells, and activated dendritic cells. Conversely, these patients may show LINC02446 down-regulation, which is associated with greater abundance of activated NK cells, M1 macrophages and CD8+ T cells. The abundance of activated dendritic cells increases as tumors grow ${ }^{[22]}$. Higher numbers of Tregs can bypass the tumor's ability to evade immune responses $^{[23]}$. Our results suggest that the expression of AL117382.2 and LINC02446 may be coordinated in a way that strengthens antitumor immunity in patients with low scores on the 20-IncRNA signature.

Scores on the 20-IncRNA signature negatively correlated with several indicators of ICl efficacy. The Teffector and interferon- $\gamma$ gene signature is associated with activated T cells, immune cytolytic activity, and interferon- $y$ expression, and the genes show patterns of co-expression in cancer patients. Immune checkpoint inhibitors of CTLA4, PDCD1 and CD274 have shown great promise in treating various malignancies ${ }^{[24,25]}$. These results further support the usefulness of the IncRNA signature for predicting the efficacy of immunotherapy. In this study, TMB was significantly negatively associated with twentyIncRNA-based signature score. And VEGF and MAPK signaling pathways were activated in COAD patients with low signature score. As in a previous study, MAPK and VEGF signaling pathways can not only affect 
cancer cell growth and tumor angiogenesis, but also affect tumor antigenicity and $T$ cell infiltration in tumors $^{[26]}$. Therefore, we speculated that the COAD patients with activation of VEGF and signaling pathway may get better treatment while receiving the immunotherapy.

There are some limitations in this study. Firstly, our results should be interpreted with caution given our reliance on retrospective data. Secondly, the model should be further developed before it can be implemented into routine clinical practice. Thirdly, further work should examine in detail how the 20 IncRNAs in the signature participate or influence immune responses to colon adenocarcinoma.

\section{Conclusions}

The 20-IncRNA signature accurately reflects TMB in colon adenocarcinoma and is associated with known indicators of ICl efficacy and preexisting immunity in such patients. These results suggest that the signature may be a novel predictor of the efficacy of ICI therapy against colon adenocarcinoma.

\section{Declarations}

\section{Conflict of Interest}

The authors declare that the research was conducted in the absence of any commercial or financial relationships that could be construed as a potential conflict of interest.

\section{Funding}

This research did not receive any specific grants from funding agencies in the public, commercial, or notfor-profit sectors.

\section{Author Contributions}

Qian Li, Ziyu Liu, Lianying Ge and Yan Lin designed and coordinated the study, and prepared the manuscript. Rong Liang, Ziqin He, Xing Gao, Jinyan Zhang provided assistance in the design of the study and participated in manuscript preparation. Jiazhou Ye, Min Luo and Yongqiang Li participated in data gathering. All authors have read and approved the content of the manuscript.

\section{Data Availability Statement}

The original contributions presented in the study are included in the article/supplementary material, further inquiries can be directed to the corresponding authors.

\section{References}


1. Torre LA, Bray F, Siegel RL, Ferlay J, Lortet-Tieulent J, Jemal A. Global cancer statistics, 2012. CA Cancer J Clin. 2015;65(2):87-108.

2. Li Y, Liang L, Dai W, et al. Prognostic impact of programed cell death-1 (PD-1) and PD-ligand 1 (PDL1) expression in cancer cells and tumor infiltrating lymphocytes in colorectal cancer. Mol Cancer. 2016;15(1):55.

3. Büttner R, Longshore JW, López-Ríos F, et al. Implementing TMB measurement in clinical practice: considerations on assay requirements. ESMO Open. 2019;4(1):e000442.

4. Büttner R, Gosney JR, Skov BG, et al. Programmed Death-Ligand 1 Immunohistochemistry Testing: A Review of Analytical Assays and Clinical Implementation in Non-Small-Cell Lung Cancer. J Clin Oncol. 2017;35(34):3867-76.

5. Gibney GT, Weiner LM, Atkins MB. Predictive biomarkers for checkpoint inhibitor-based immunotherapy. Lancet Oncol. 2016;17(12):e542-51.

6. Hellmann MD, Ciuleanu TE, Pluzanski A, et al. Nivolumab plus Ipilimumab in Lung Cancer with a High Tumor Mutational Burden. N Engl J Med. 2018;378(22):2093-104.

7. Riaz N, Havel JJ, Makarov V, et al. Tumor and Microenvironment Evolution during Immunotherapy with Nivolumab. Cell. 2017;171(4):934-49.e16.

8. Jing H, Qu X, Liu L, Xia H. A Novel Long Noncoding RNA (IncRNA), LL22NC03-N64E9.1, Promotes the Proliferation of Lung Cancer Cells and is a Potential Prognostic Molecular Biomarker for Lung Cancer. Med Sci Monit. 2018;24:4317-23.

9. Terzić J, Grivennikov S, Karin E, Karin M. Inflammation and colon cancer. Gastroenterology. 2010;138(6):2101-14.e5.

10. Vogelstein B, Fearon ER, Hamilton SR, et al. Genetic alterations during colorectal-tumor development. N Engl J Med. 1988;319(9):525-32.

11. O'Connell JB, Maggard MA, Ko CY. Colon cancer survival rates with the new American Joint Committee on Cancer sixth edition staging. J Natl Cancer Inst. 2004;96(19):1420-5.

12. Siebenkäs C, Chiappinelli KB, Guzzetta AA, et al. Correction: Inhibiting DNA methylation activates cancer testis antigens and expression of the antigen processing and presentation machinery in colon and ovarian cancer cells. PLoS One. 2020;15(12):e0243944.

13. Lu XF, Xia XF, Chen G. [Effect of peroxisome proliferator-activated receptor-y coactivator-1a on metastasis and anoikis resistance in colorectal cancer]. Zhonghua Zhong Liu Za Zhi. 2016;38(7):499-503.

14. Yang LC, Lai CY, Hsieh CC, Lin WC. Natural killer cell-mediated anticancer effects of an arabinogalactan derived from rice hull in CT26 colon cancer-bearing mice. Int J Biol Macromol. 2019;124:368-76.

15. Strickland KC, Howitt BE, Shukla SA, et al. Association and prognostic significance of BRCA1/2mutation status with neoantigen load, number of tumor-infiltrating lymphocytes and expression of PD-1/PD-L1 in high grade serous ovarian cancer. Oncotarget. 2016;7(12):13587-98. 
16. Thomas A, Routh ED, Pullikuth A, et al. Tumor mutational burden is a determinant of immunemediated survival in breast cancer. Oncoimmunology. 2018;7(10):e1490854.

17. Bindea G, Mlecnik B, Tosolini M, et al. Spatiotemporal dynamics of intratumoral immune cells reveal the immune landscape in human cancer. Immunity. 2013;39(4):782-95.

18. Poznanski SM, Barra NG, Ashkar AA, Schertzer JD. Immunometabolism of T cells and NK cells: metabolic control of effector and regulatory function. Inflamm Res. 2018;67(10):813-28.

19. Nera KP, Kyläniemi MK, Lassila O. Regulation of B Cell to Plasma Cell Transition within the Follicular B Cell Response. Scand J Immunol. 2015;82(3):225-34.

20. Novy P, Quigley M, Huang $X$, Yang Y. CD4 T cells are required for CD8 T cell survival during both primary and memory recall responses. J Immunol. 2007;179(12):8243-51.

21. Zhang R, Li F, Li H, Yu J, Ren X. The clinical significance of memory T cells and its subsets in gastric cancer. Clin Transl Oncol. 2014;16(3):257-65.

22. Zhang SC, Hu ZQ, Long JH, et al. Clinical Implications of Tumor-Infiltrating Immune Cells in Breast Cancer. J Cancer. 2019;10(24):6175-84.

23. Bézie S, Charreau B, Vimond N, et al. Human CD8+ Tregs expressing a MHC-specific CAR display enhanced suppression of human skin rejection and GVHD in NSG mice. Blood Adv. 2019;3(22):3522-38.

24. Fesnak $A D$, June $\mathrm{CH}$, Levine BL. Engineered T cells: the promise and challenges of cancer immunotherapy. Nat Rev Cancer. 2016;16(9):566-81.

25. Basile D, Garattini SK, Bonotto M, et al. Immunotherapy for colorectal cancer: where are we heading. Expert Opin Biol Ther. 2017;17(6):709-21.

26. Pitt JM, Vétizou M, Daillère R, et al. Resistance Mechanisms to Immune-Checkpoint Blockade in Cancer: Tumor-Intrinsic and -Extrinsic Factors. Immunity. 2016;44(6):1255-69.

\section{Tables}

Due to technical limitations, tables are only available as a download in the Supplemental Files section.

\section{Figures}



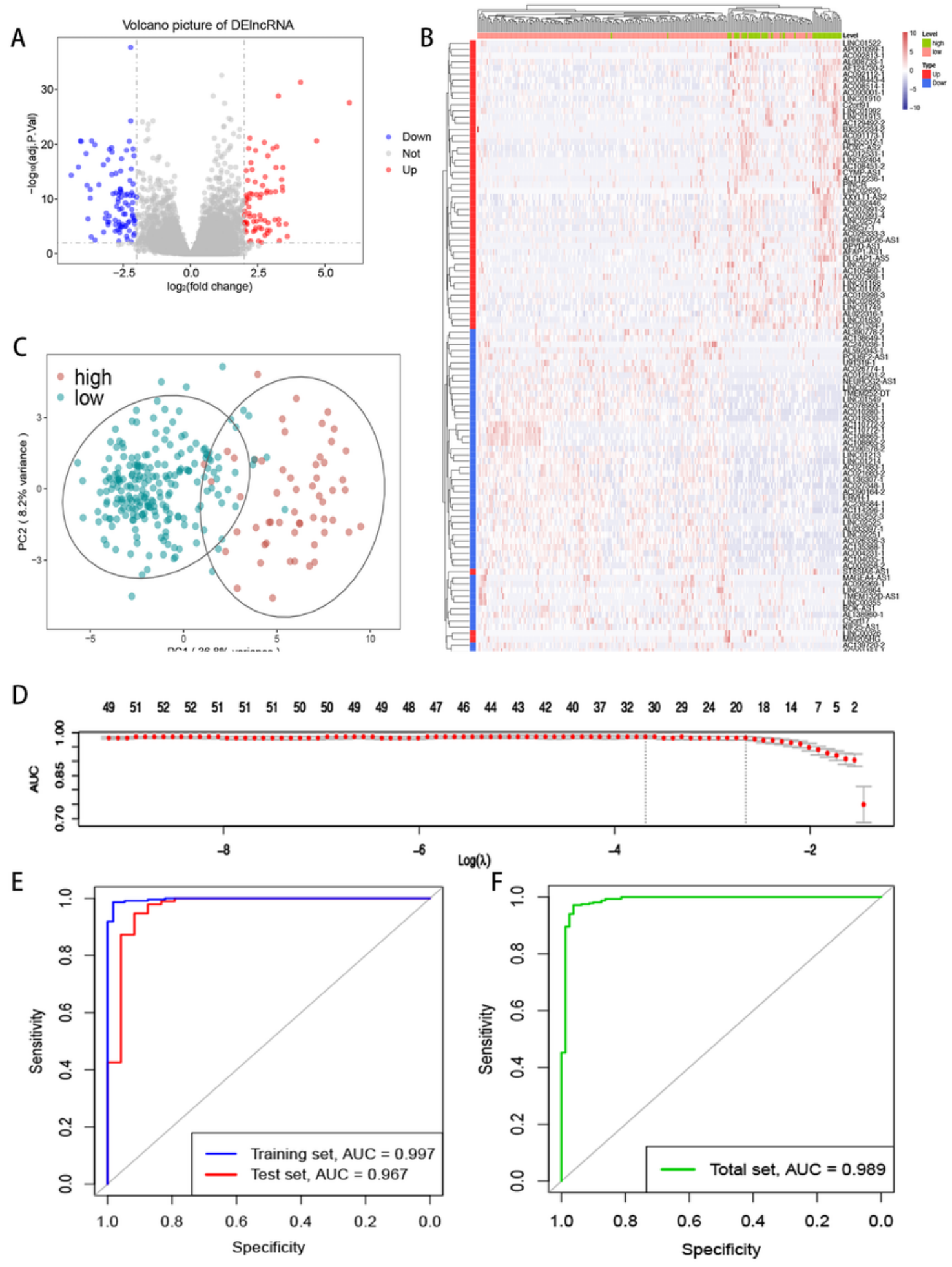

\section{Figure 1}

Long non-coding RNAs differentially expressed (DE IncRNAs) between colon adenocarcinoma tumors with low or high tumor mutation burden. (A) Volcano plot of DE IncRNAs. (B) Hierarchical clustering of the most up- and down-regulated DE IncRNAs, ranked according to log2 fold change. (C) Principal component analysis based on DE IncRNAs. 
(D) Feature selection using the absolute shrinkage and selection operator method and 10-fold crossvalidation. (E) Receiver operating characteristic curve to assess the reliability of the signature score in the training and test cohorts. (F) Receiver operating characteristic curve to assess the reliability of the signature score in the two cohorts combined.
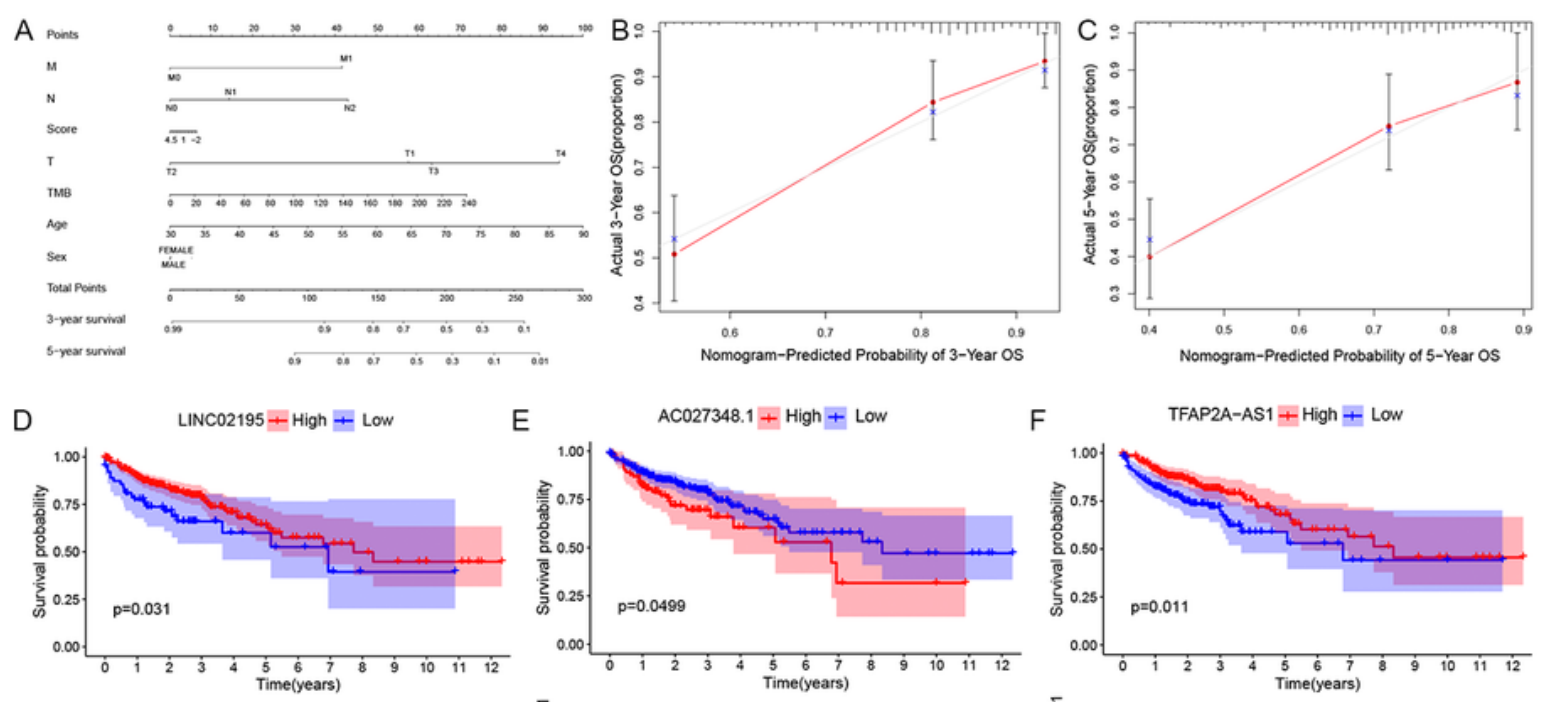

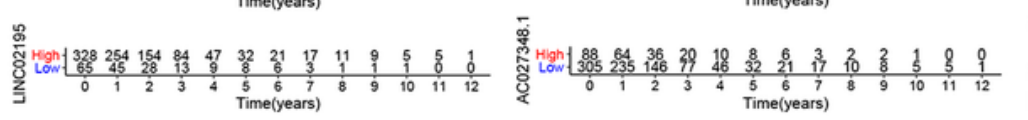
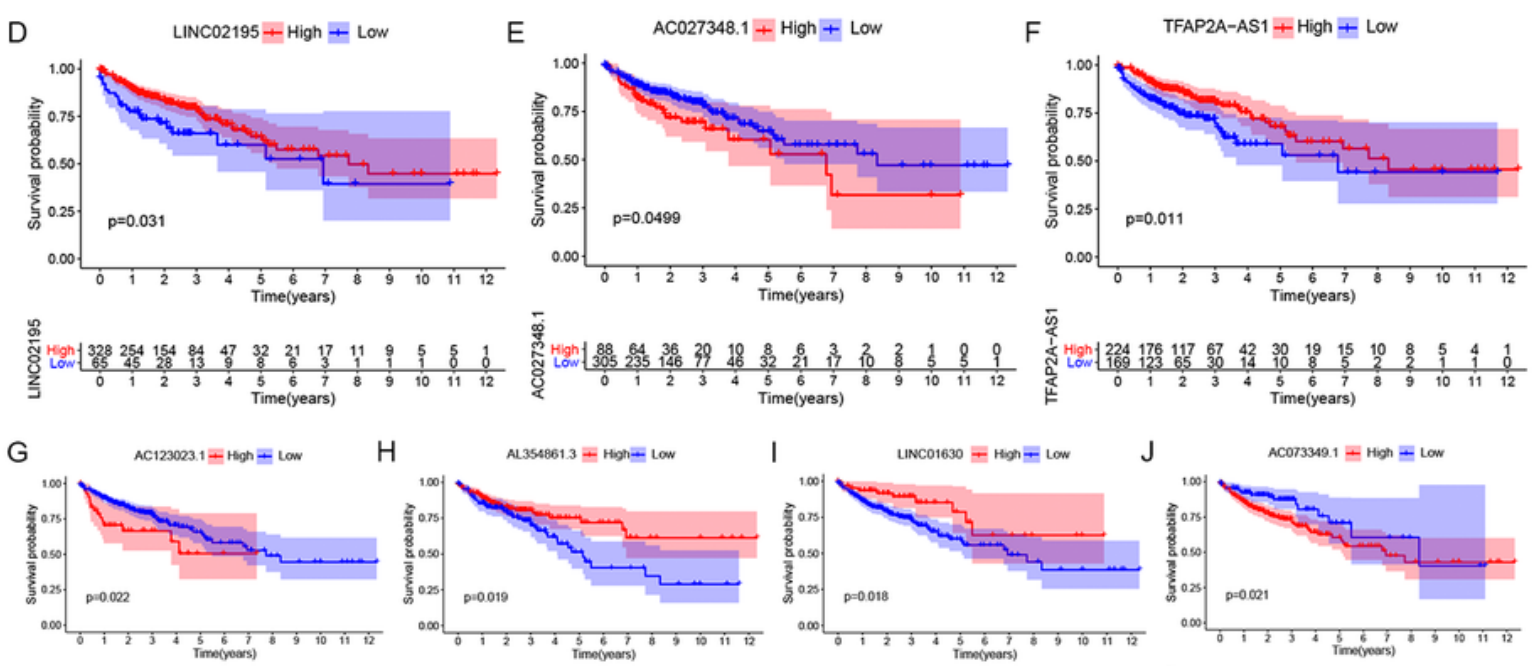

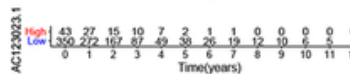

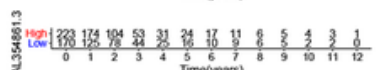

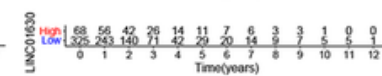

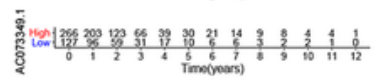

K

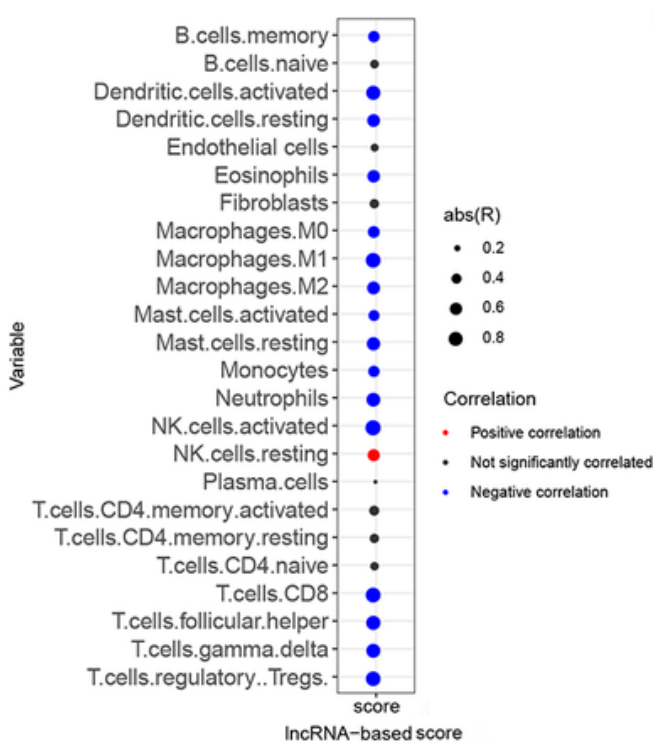

L

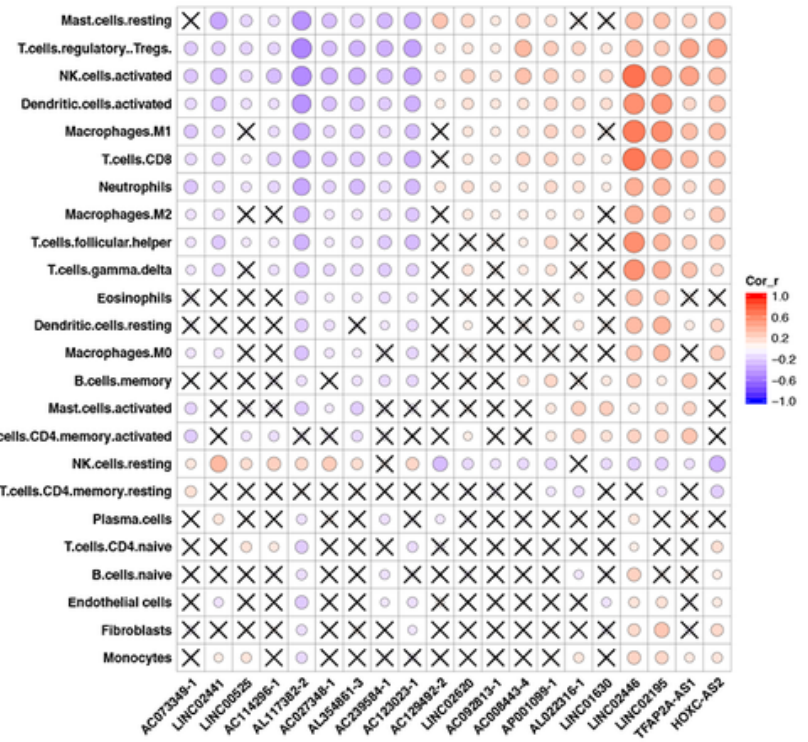

Figure 2 
Nomogram, calibration curves and survival analyses of patients with COAD. (A) Nomogram to predict overall survival (OS) at 3 years and 5 years. (B) Calibration plot for nomogram predicted and observed 3year overall survival rate. (C) Calibration plot for nomogram predicted and observed 5-year overall survival rate. The OS analyses for (D) LINC02195, (E) AC027348.1, (F) TFAP2A-AS1, (G)AC123023.1, (H)AL354861.3, (I) LINC01630, (J) AC073349.1, low-expressed and high-expressed COAD patients. All the points assigned on the top point scale for each factor are summed together to generate total point score. The total point score is projected on the bottom scales to determine probability of 3- or 5-year survival rate in an individual. The red line represents the actual observed 3- or 5-year survival rate, the grey line represents the observed of that. Correlations between scores on the 20-IncRNA signature and TILs were assessed using $(\mathrm{K})$ a bubble heat map and $(\mathrm{L})$ correlation heat map. 

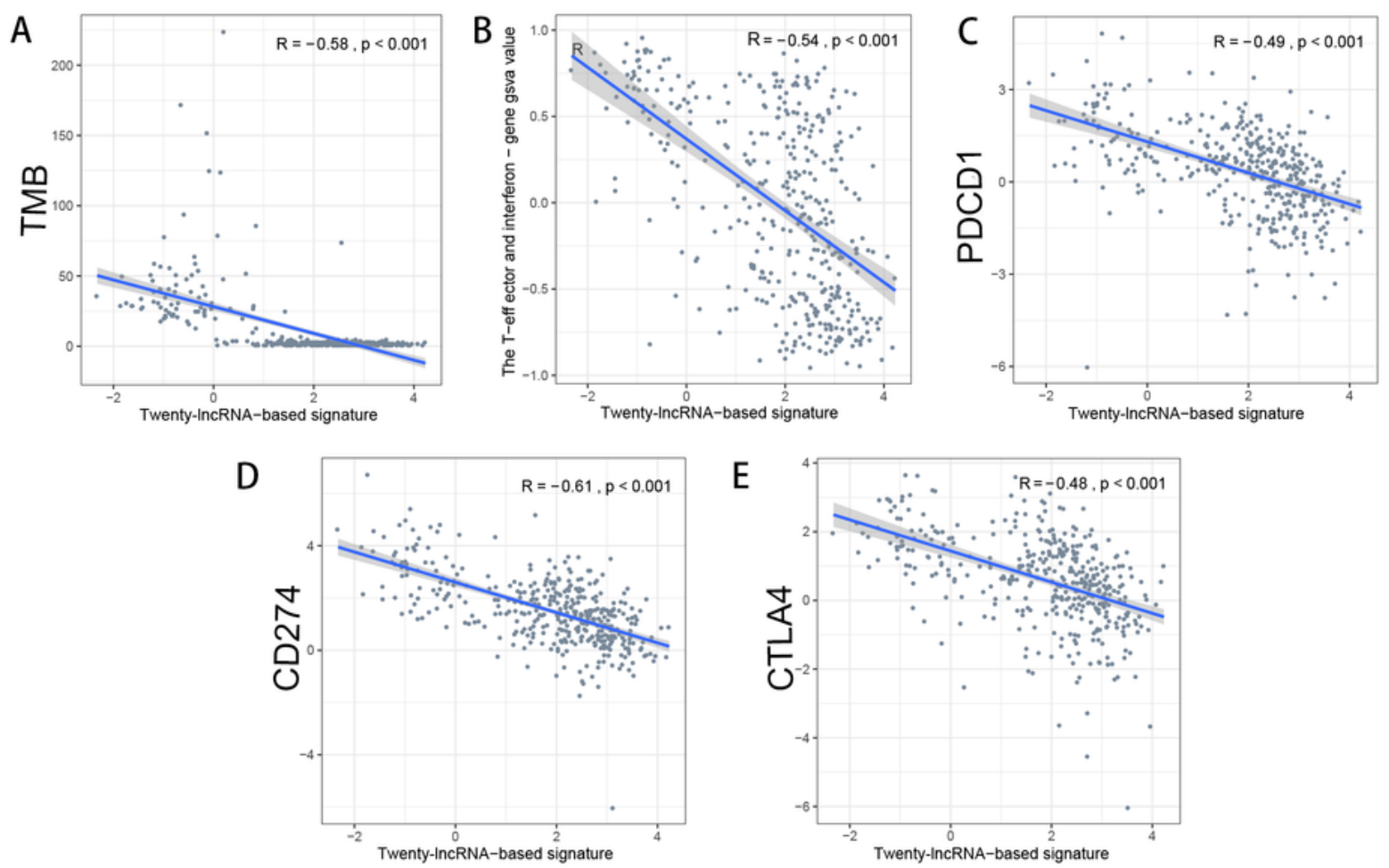

Significant variation of top 30 KEGG pathways

$\mathrm{F}$

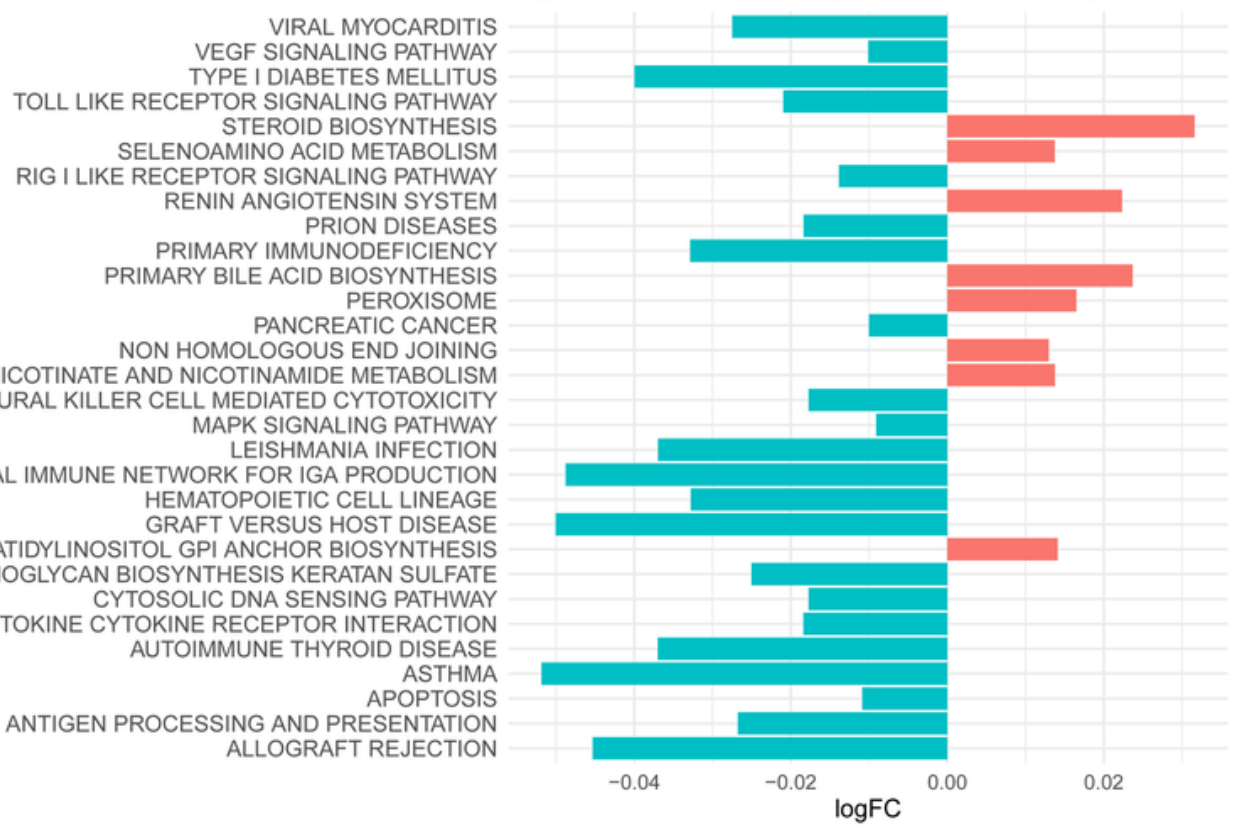

Type

Activer in high signature score

Activer in low signature score

\section{Figure 3}

Association of the 20-IncRNA signature with indicators of immune checkpoint inhibitor efficacy against colon adenocarcinoma. Correlations of the signature score with (A) TMB, (B) T-effector and interferon- $\gamma$ gene signature as well as expression of (C) PDCD1, (D) CD274 and (E) CTLA4 were examined. (F) Pathways differentially activated between colon adenocarcinoma tumors showing low or high scores on 
the 20-IncRNA signature. Only the 30 pathways showing the most statistically significant differences (based on $\mathrm{P}$ value) are shown.

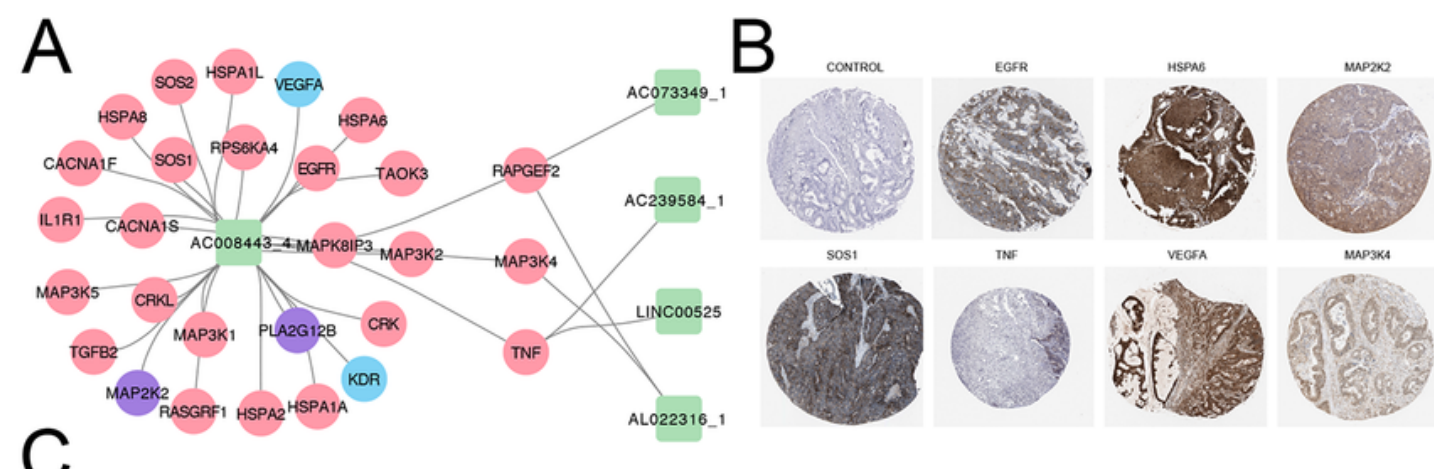

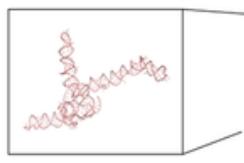

$\mathrm{AC} 008443.4$

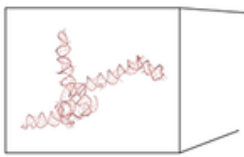

AC008443.4

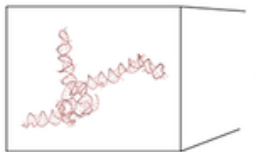

AC008443.4

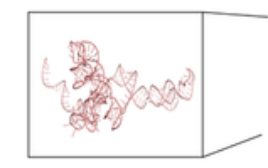

$D^{\text {M.0202361 }}$

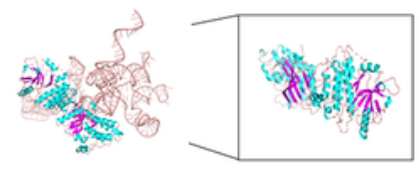

Etotal: $-373.31 \mathrm{kj} / \mathrm{mol}$

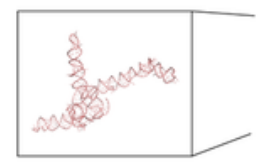

AC008443.4

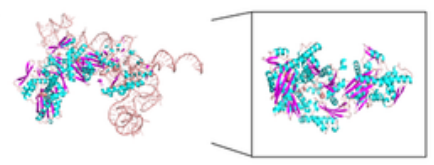

Etotal: $-209.80 \mathrm{kj} / \mathrm{mol}$

HSPA6

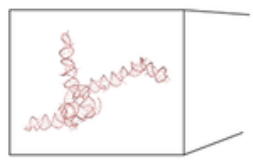

AC008443.4

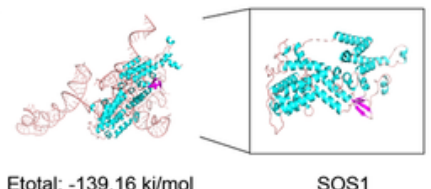

Etotal: $-448.70 \mathrm{kj} / \mathrm{mol}$

MAP2K2

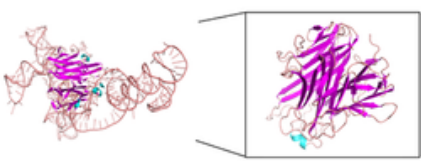

TNF

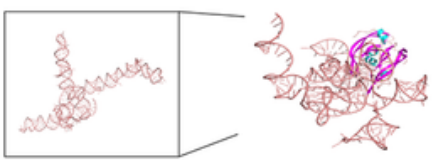

AC008443.4

Etotal: $-646.63 \mathrm{kj} / \mathrm{mol}$

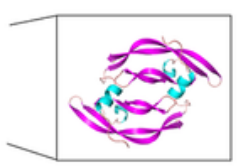

Etotal: $-552.37 \mathrm{kj} / \mathrm{mol}$

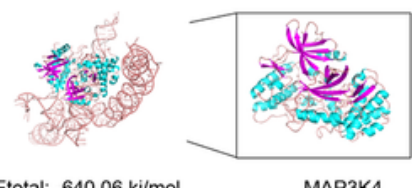

MAP3K4

Etotal: $-640.06 \mathrm{kj} / \mathrm{mo}$

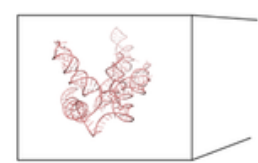

LINC00525

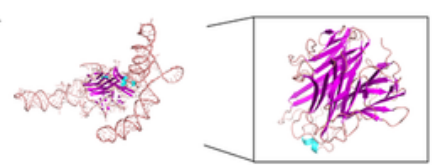

Etotal: $-830.67 \mathrm{kj} / \mathrm{mol}$

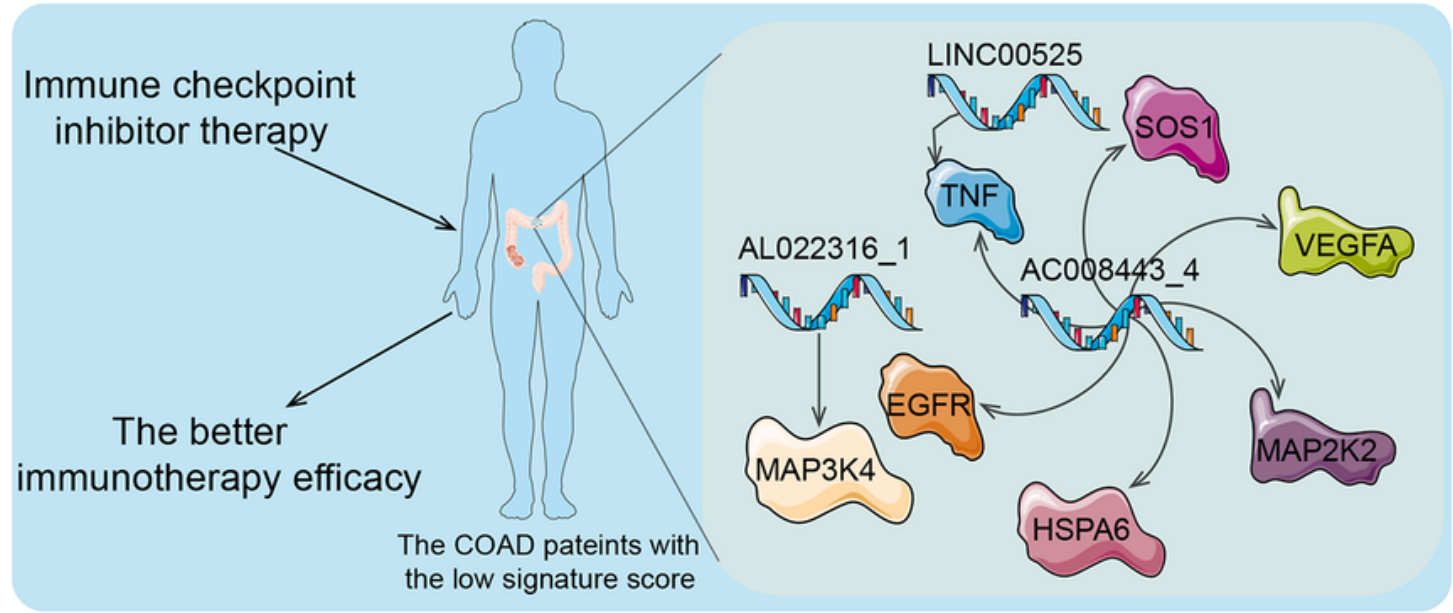

Figure 4

The signature IncRNA-protein interaction and the proposed mechanism. A. The interaction network. The circle represents protein. The round rectangle represents IncRNA. The pink represents proteins encoded by 
genes in MAPK signaling pathway. The light blue represents proteins encoded by genes in VEGF signaling pathway. The purple represents the proteins encoded by common genes in VEGF and MAPK signaling pathway. B. Representative images of immunohistochemical staining from COAD patients and control. Showed high expression of target proteins in COAD tissues. C. The molecular docking of characteristic IncRNAs and target proteins, and the energy less than 0 represents the potential for docking. D. The proposed mechanism.
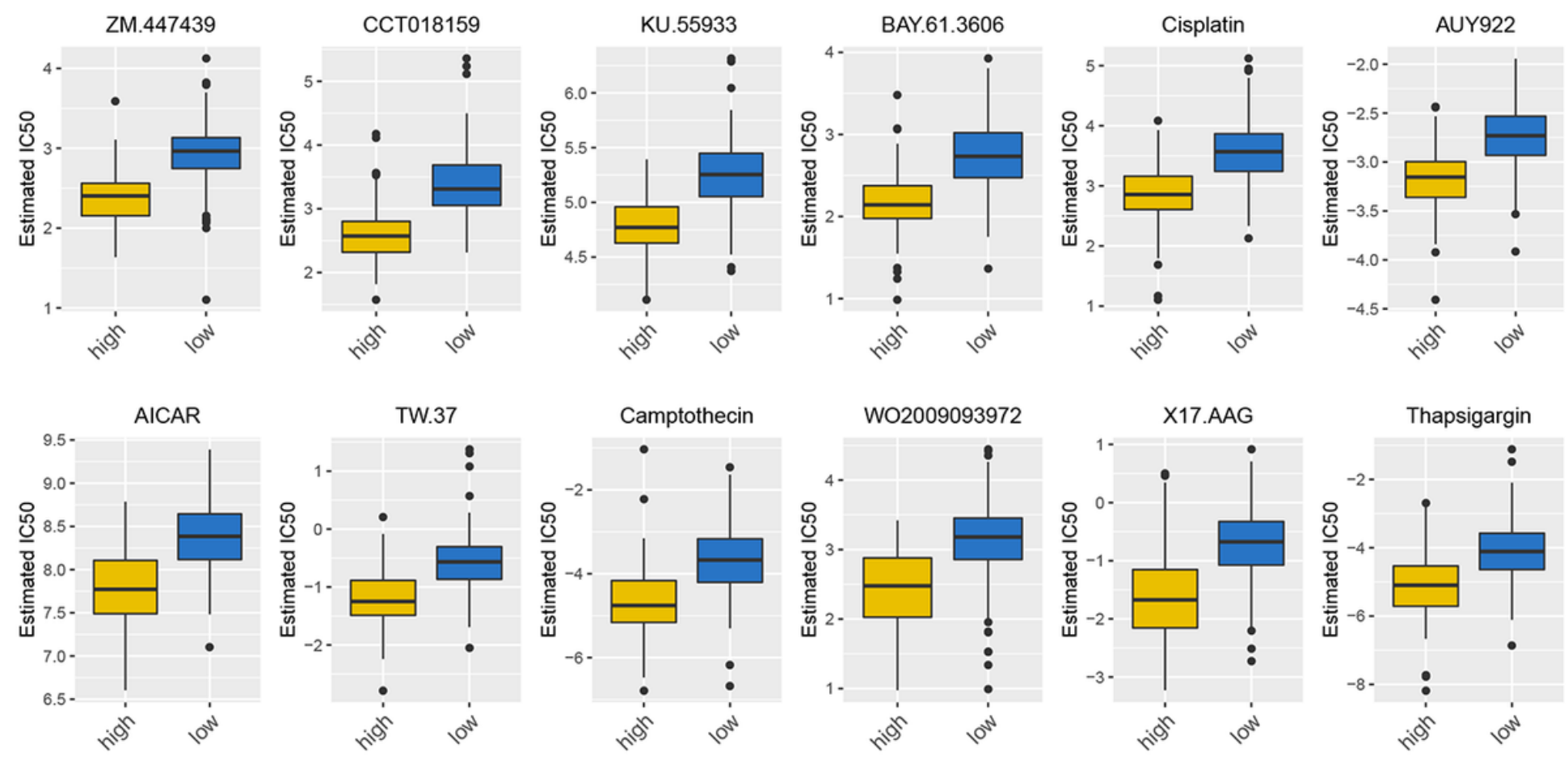

Figure 5

Drug response characteristics based on TMB related DE IncRNAs.

\section{Supplementary Files}

This is a list of supplementary files associated with this preprint. Click to download.

- Table1.xlsx

- Table2.xlsx

- Table3.xlsx

- SupplementaryMaterialsandMethods.docx

- figures1.png 